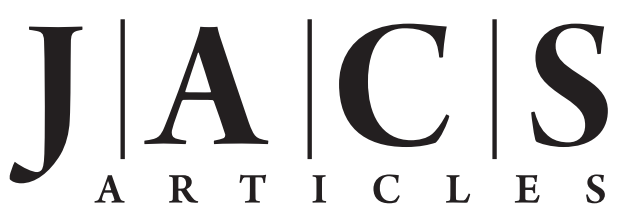

Published on Web 07/20/2010

\title{
Dynamics Characterization of Fully Hydrated Bacterial Cell Walls by Solid-State NMR: Evidence for Cooperative Binding of Metal lons
}

\author{
Thomas Kern, ${ }^{\dagger, \perp}$ Mathilde Giffard, ${ }^{\ddagger, \perp}$ Sabine Hediger, ${ }^{\ddagger}$ Ana Amoroso, ${ }^{\S}$ \\ Cécile Giustini, ${ }^{\dagger}$ Nhat Khai Bui," Bernard Joris, ${ }^{\S}$ Catherine Bougault, ${ }^{\dagger}$ \\ Waldemar Vollmer," and Jean-Pierre Simorre ${ }^{*, \dagger}$
}

Institut de Biologie Structurale, UMR5075 (CEA/CNRS/UJF), 38027 Grenoble, France, Laboratoire de Chimie Inorganique et Biologique, UMR-E3 (CEA/UJF), FRE3200 (CEA/CNRS), INAC, CEA, 38054 Grenoble, France, Centre d'ingénierie des protéines, Institut de Chimie B6A, Université de Liège, Sart-Tilman, B4000 Liège, Belgium, and Centre for Bacterial Cell Biology, Institute for Cell and Molecular Biosciences, Newcastle University, Richardson Road, Newcastle upon Tyne NE2 4AX, United Kingdom

Received May 25, 2010; E-mail: jean-pierre.simorre @ibs.fr

\begin{abstract}
The bacterial cell wall maintains a cell's integrity while allowing growth and division. It is made up of peptidoglycan (PG), a biopolymer forming a multigigadalton bag-like structure, and, additionally in Gram-positive bacteria, of covalently linked anionic polymers collectively called teichoic acids. These anionic polymers are thought to play important roles in host-cell adhesion, inflammation, and immune activation. In this Article, we compare the flexibility and the organization of peptidoglycans from Gram-negative bacteria (E. coli) with its counterpart from different Gram-positive bacteria using solid-state nuclear magnetic resonance spectroscopy (NMR) under magic-angle sample spinning (MAS). The NMR fingerprints suggest an identical local conformation of the PG in all of these bacterial species. Dynamics in the peptidoglycan network decreases from $E$. coli to $B$. subtilis and from $B$. subtilis to $S$. aureus and correlates mainly with the degree of peptide cross-linkage. For intact bacterial cells and isolated cell walls, we show that ${ }^{31} \mathrm{P}$ solid-state NMR is particularly well adapted to characterize and differentiate wall teichoic acids of different species. We have further observed complexation with divalent ions, highlighting an important structural aspect of Gram-positive cell wall architecture. We propose a new model for the interaction of divalent cations with both wall teichoic acids and carbonyl groups of peptidoglycan.
\end{abstract}

\section{Introduction}

Peptidoglycan is the major structural component of the cell envelope of most Gram-positive and Gram-negative bacteria. ${ }^{1}$ It encases the cytoplasmic membrane, giving the cell its shape and protecting it from lysis due to turgor. Being crucial for the bacteria's survival, it is the key target of antibacterial treatment. The immune system also recognizes peptidoglycan fragments released from bacterial cells provoking the innate immune response. $^{2}$

The primary structure of peptidoglycan consists of glycan strands of alternating $N$-acetylmuramic acid (MurNAc) and $\mathrm{N}$-acetylglucosamine (GlcNAc) residues cross-linked by short peptides that are attached to the lactyl group of MurNAc (Figure 1a). The glycan strand length varies depending on the species and growth conditions. ${ }^{3-6}$ Within given species, structural variations in the peptidoglycan can also occur; for example, in

\footnotetext{
$\dagger$ Institut de Biologie Structurale.

* Laboratoire de Chimie Inorganique et Biologique.

§niversité de Liège.

"Newcastle University.

${ }^{\perp}$ These authors contributed equally.

(1) Vollmer, W.; Blanot, D.; de Pedro, M. A. FEMS Microbiol. Rev. 2008, 32, 149-167.

(2) Chaput, C.; Boneca, I. G. Microbes Infect. 2007, 9, 637-647.
}

B. subtilis amidation of meso-diaminopimelic acid (L,D- or meso$\mathrm{A}_{2} \mathrm{pm}$ ) occurs after the action of Mur ligases (i.e., the enzymes responsible for the synthesis of the peptide in the peptidoglycan precursor) to variable extent depending on the growth conditions. ${ }^{7,8}$ The elementary repeating units of the peptidoglycan in E. coli, B. subtilis, and S. aureus are shown in Figure 1b,c. Peptide cross-linking most often occurs between the amino acid at position 3 [meso-A $\mathrm{A}_{2} \mathrm{pm}$ for E. coli and B. subtilis, L-Lys $\left(\mathrm{Gly}_{5}\right)$ for $S$. aureus] of one peptide strand and the D-Ala at position 4 of another peptide strand. The cross-link is either direct (in $E$. coli and B. subtilis) or through an interpeptide-bridge such as the pentaglycine fragment in $S$. aureus (Figure 1b,c). The degree of cross-linking is species as well as growth-phase (exponential

(3) Harz, H.; Burgdorf, K.; Höltje, J. Anal. Biochem. 1990, 190, 120128.

(4) Vollmer, W.; Seligman, S. J. Trends Microbiol. 2010, 18, 59-66.

(5) Glauner, B.; Höltje, J.; Schwarz, U. J. Biol. Chem. 1988, 263, 1008810095.

(6) Hayhurst, E. J.; Kailas, L.; Hobbs, J. K.; Foster, S. J. Proc. Natl. Acad. Sci. U.S.A. 2008, 105, 14603-14608.

(7) Warth, A. D.; Strominger, J. L. Biochemistry 1971, 10, 4349-4358.

(8) Atrih, A.; Bacher, G.; Allmaier, G.; Williamson, M. P.; Foster, S. J. J. Bacteriol. 1999, 181, 3956-3966. 
(a)

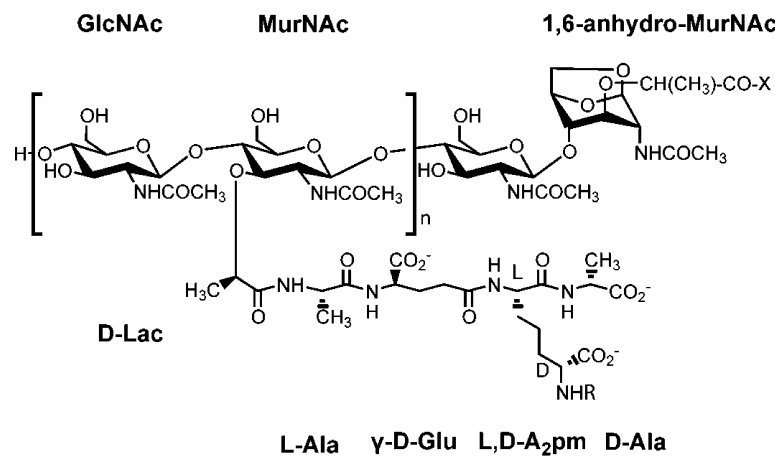

(b)

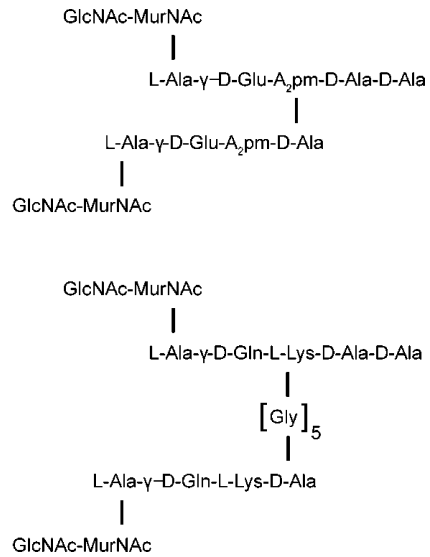

Figure 1. Repeating unit of the peptidoglycan of (a) E. coli or B. subtilis, and schematic representation of different peptide cross-links found in the peptidoglycan networks of (b) E. coli, B. subtilis, and (c) S. aureus. X indicates a peptide linked to the lactyl group of 1,6-anhydro-MurNAc and R the cross-linked peptide attached to a neighboring glycan strand.
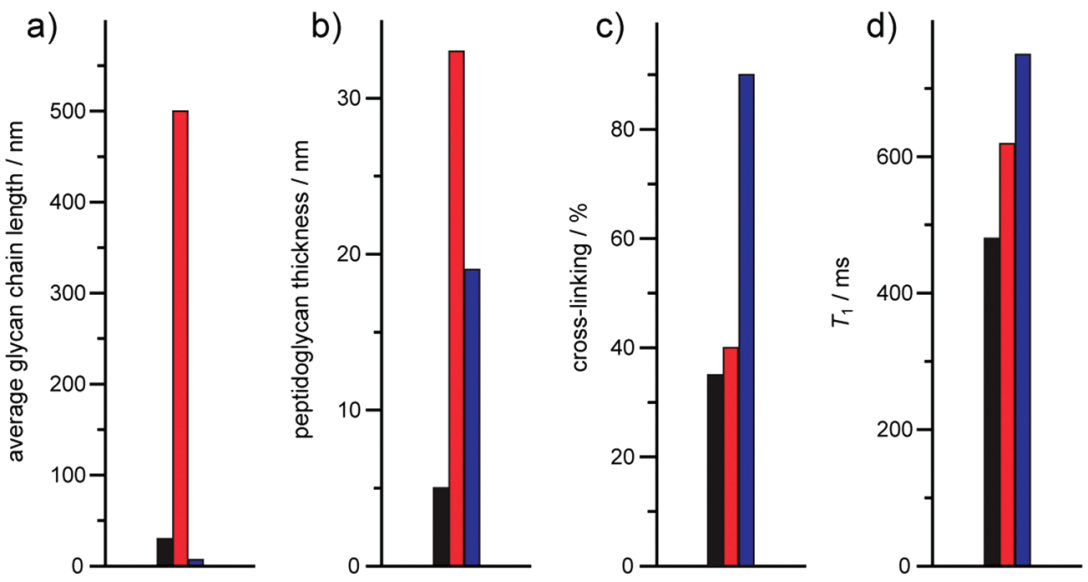

Figure 2. Variation of peptidoglycan global structural parameters [(a) chain length, (b) peptidoglycan thickness, and (c) percentage of peptidoglycan crosslinking] within three bacterial species: E. coli (black), B. subtilis (red), and S. aureus (blue). $T_{1}$ relaxation time-constants shown in panel (d) correspond to the average of values measured on all ${ }^{13} \mathrm{C}$ nuclei (see Results).

versus stationary phase) dependent. ${ }^{1,9}$ Variations of global peptidoglycan parameters (glycan chain length, peptidoglycan thickness, and degree of cross-linking) are shown in Figure 2 for E. coli, B. subtilis, and S. aureus species.

In most Gram-positive bacteria, a network of anionic cell wall polymers, called teichoic acids (TAs), is present in the cell envelope. Wall teichoic acids (WTAs) are covalently bound to the $N$-acetylmuramic acid of the peptidoglycan by a phosphate group, whereas lipoteichoic acids (LTAs) are anchored by a glycolipid to the cytoplasmic membrane. In Gram-positive bacteria lacking capsule and S-layer, like B. subtilis, peptidoglycan and TAs are at the interface between the cell and its environment. The polyionic network formed by the TAs was proposed to have many diverse roles. Of these, the best known relates to the regulation of metal cation homeostasis and the ion trafficking into and out of the cytoplasm. ${ }^{10}$ TAs are also implicated in cell adhesion mechanisms through their interaction with mammalian receptors ${ }^{11}$ and play a key role in nasal

(9) de Jonge, B. L.; Chang, Y. S.; Gage, D.; Tomasz, A. J. Biol. Chem. 1992, 267, 11255-11259.

(10) Neuhaus, F. C.; Baddiley, J. Microbiol. Mol. Biol. Rev. 2003, 67, 686723.

(11) Peiser, L.; Mukhopadhyay, S.; Gordon, S. Curr. Opin. Immunol. 2002, 14, 123-128. colonization by $S$. aureus. ${ }^{12}$ It has also been shown that TAs act in the modulation of interactions between the cell surface and endogenous cationic antimicrobial peptides (CAMPs), ${ }^{13}$ as well as in the formation of biofilms. ${ }^{14}$ Most WTAs and LTAs are composed of a linkage unit followed by linear poly(ribitolphosphate) or poly-(glycerolphosphate) chains (Figure 3). ${ }^{15}$ Several substituents are frequently present on the repeating units where they modulate the TA activities.

Although the chemical composition of the peptidoglycan and teichoic acids of a large number of bacteria has been determined, very little is known about their three-dimensional structure. The true architecture of the cell wall remains poorly determined, primarily because of its large size (up to $3 \times 10^{6} \mathrm{kDa}$ for $E$. coli) and its noncrystalline state. Biomolecular solid-state nuclear magnetic resonance spectroscopy (SSNMR) has made huge progress in the last years, becoming a promising method for the structural characterization of noncrystalline biopolymers. ${ }^{16}$ Currently, only a few SSNMR studies have been performed on

(12) Weidenmaier, C.; Kokai-Kun, J. F.; Kristian, S. A.; Chanturiya, T.; Kalbacher, H.; Gross, M.; Nicholson, G.; Neumeister, B.; Mond, J. J.; Peschel, A. Nat. Med. 2004, 10, 243-245.

(13) Peschel, A.; Sahl, H. Nat. Rev. Microbiol. 2006, 4, 529-536.

(14) Weidenmaier, C.; Peschel, A. Nat. Rev. Microbiol. 2008, 6, 276-287.

(15) Rahman, O.; Dover, L. G.; Sutcliffe, I. C. Trends Microbiol. 2009, 17, 219-225.

(16) McDermott, A. E. Annu. Rev. Biophys. 2009, 38, 385-403. 

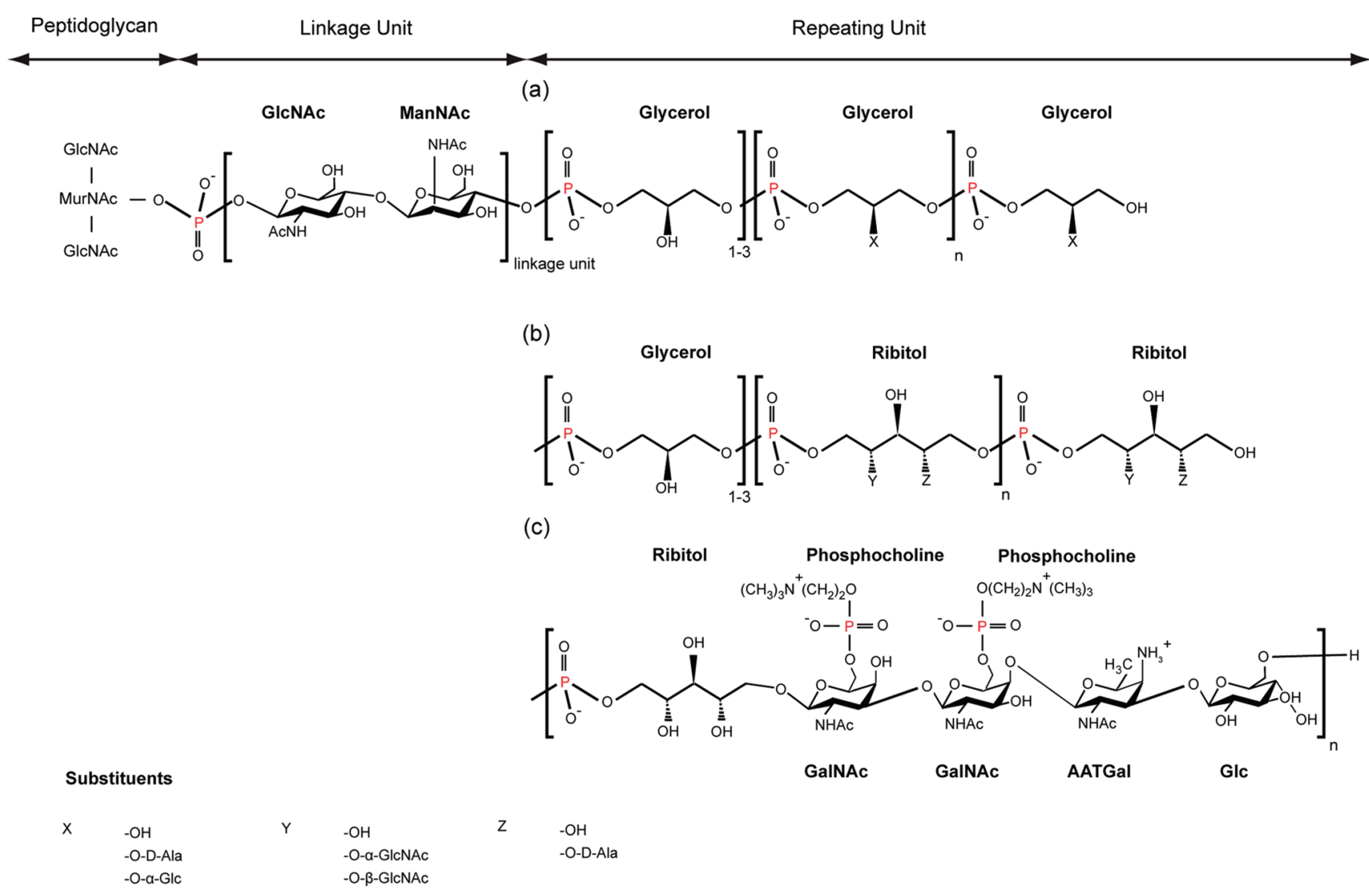

Figure 3. Chemical structure of WTAs in (a) B. subtilis 168, (b) S. aureus, and (c) S. pneumoniae R6. WTAs are covalently bound at position 6 of the $\mathrm{N}$-acetylmuramic acid of the glycan strand. Possible substituents $\mathrm{X}, \mathrm{Y}$, and $\mathrm{Z}$ of glycerol or ribitol are detailed at the bottom of the figure.

bacterial cell wall samples ${ }^{17-19}$ and mainly on freeze-dried samples. Recently, we showed that working on fully hydrated samples leads to well-resolved NMR spectra of astonishing good quality. ${ }^{19}$ Therefore, a site-specific characterization of the dynamics and local structure of cell walls from different bacterial species becomes possible. The present work focuses on $B$. subtilis and $S$. aureus. A combination of solid-state ${ }^{13} \mathrm{C}-{ }^{13} \mathrm{C}$ correlation experiments and ${ }^{31} \mathrm{P}$ NMR spectroscopy proved to provide key structural as well as metal-binding information. Our approach opens new ways to analyze entire bacterial cell walls under fully hydrated conditions.

\section{Results}

Characterization of the Peptidoglycan from B. subtilis 168 and $S$. aureus. SQ/SQ ${ }^{13} \mathrm{C}-{ }^{13} \mathrm{C} J$-correlation experiments were systematically recorded on both cell wall and peptidoglycan samples from B. subtilis and $S$. aureus. Overlay of the corresponding NMR spectra showed that the chemical shift of the resonances from the peptidoglycan remained unaffected by the presence of the WTAs. This comparison confirmed that the peptidoglycan structure is not altered by the removal of the teichoic acids in either strain. ${ }^{13} \mathrm{C}$ spectra of the peptidoglycan of Gram-positive bacteria were further compared to the one measured on the E. coli Gram-negative bacterial model. Figure

(17) Kim, S. J.; Cegelski, L.; Preobrazhenskaya, M.; Schaefer, J. Biochemistry 2006, 45, 5235-5250.

(18) Kim, S. J.; Cegelski, L.; Stueber, D.; Singh, M.; Dietrich, E.; Tanaka, K. S. E.; Parr, T. R. J.; Far, A. R.; Schaefer, J. J. Mol. Biol. 2008, 377, 281-293.

(19) Kern, T.; Hediger, S.; Müller, P.; Giustini, C.; Joris, B.; Bougault, C.; Vollmer, W.; Simorre, J. J. Am. Chem. Soc. 2008, 130, 56185619. 4a details this comparison for the carbonyl-to-aliphatic region of the ${ }^{13} \mathrm{C}-{ }^{13} \mathrm{C}$ correlation spectra of E. coli, B. subtilis, and $S$. aureus, and reveals a very limited number of differences. In the case of $B$. subtilis, additional peaks are observed in the COto- $\mathrm{C}_{\alpha}$ or $\mathrm{CO}-$ to- $\mathrm{C}_{\gamma}$ or $\mathrm{CO}-$ to- $\mathrm{C}_{\varepsilon}$ chemical shift regions corresponding to glutamic acid and meso- $\mathrm{A}_{2} \mathrm{pm}$ residues. This is consistent with the formerly described partial amidation of meso$\mathrm{A}_{2} \mathrm{pm}$ in the peptidoglycan of B. subtilis. ${ }^{7,8}$ For $S$. aureus, the most significant differences as compared to $E$. coli arise from the intense signal assigned to the glycine residues of the pentaglycine interpeptide bridge, from the presence of L-lysine instead of $m e s o-\mathrm{A}_{2} \mathrm{pm}$ in the peptide strands, and from the chemical shift variations of the glutamic acid CO-to- $\mathrm{C}_{\alpha}$ correlations due to a significant amidation of the Glu residue into Gln.

We presented recently dipolar-based correlation NMR experiments, which revealed that glycan chains are more rigid than the peptide stems in E. coli. ${ }^{19}$ Using similar experiments (not shown), the same finding was found for $B$. subtilis and $S$. aureus. To compare in detail the local dynamics of the peptidoglycan network of Gram-negative and Gram-positive bacteria, carbon longitudinal relaxation time-constants $T_{1}$ were measured for peptidoglycans from E. coli, B. subtilis, and $S$. aureus using inversion-recovery experiments (Figure $4 \mathrm{~b}$ ). $T_{1}$ time-constants are sensitive to dynamics in the $\mathrm{MHz}$ range, with shorter $T_{1}$ values indicating a higher flexibility as shown by its temperature dependence (see Figure S1 in the Supporting Information). These relaxation time-constants were then compared to the $T_{1}$ values obtained for peptidoglycan isolated from E. coli. ${ }^{19}$ These measurements show a homogeneous increase of the $T_{1}$ values for all resonances of the peptidoglycan of $B$. subtilis as compared to $E$. coli and of $S$. aureus as compared to $B$. subtilis. This points 

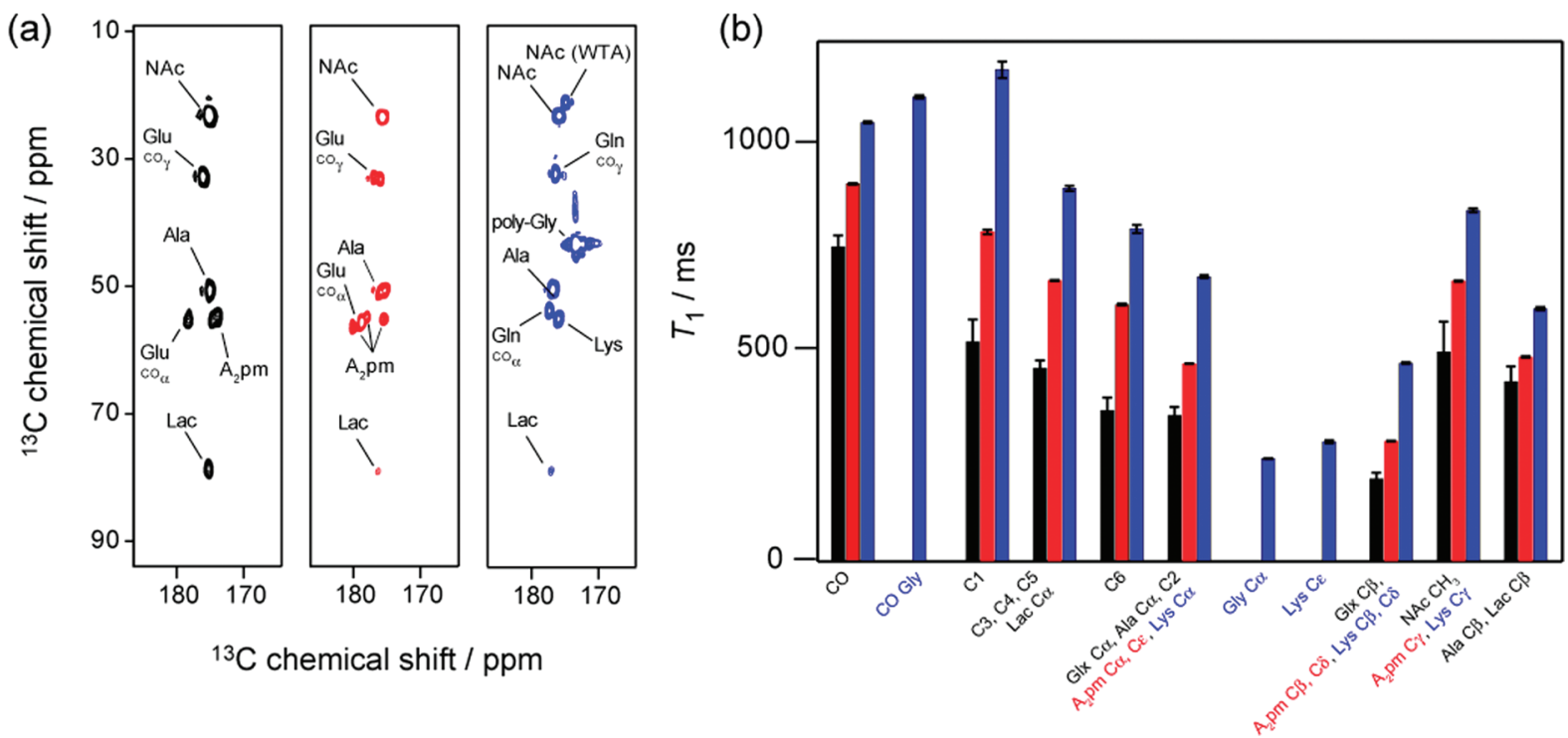

Figure 4. Comparison of (a) the carbonyl-to-aliphatic region of the ${ }^{13} \mathrm{C}-{ }^{13} \mathrm{C} \mathrm{J}$-correlation spectra and of (b) the ${ }^{13} \mathrm{C}$ longitudinal relaxation time-constants $T_{1}$ collected on a $400.13 \mathrm{MHz}$ Bruker spectrometer on cell wall samples from E. coli (black), B. subtilis 168 (red), and S. aureus (blue). Relaxation timeconstants $T_{1}$ reported in part (b) were measured on WTA-free samples.

out a global decrease of the molecular flexibility of the peptidoglycan in the following order: E. coli $>$ B. subtilis $>S$. aureus. Hence, the peptidoglycan of $S$. aureus is the most rigid of the three peptidoglycans studied in this Article.

Characterization of the Wall Teichoic Acids. Resonances of WTAs have been readily identified by comparing NMR ${ }^{13} \mathrm{C}-{ }^{13} \mathrm{C}$ correlation spectra recorded on sacculi with and without WTAs. Using a combination of characteristic correlation patterns and key chemical shifts, individual resonances of WTAs could be unambiguously assigned (Table S1). Their chemical shifts are comparable to ${ }^{13} \mathrm{C}$ data published on extracted lipoteichoic acids or cell wall polysaccharide fragments ${ }^{20}$ studied by liquid-state NMR. Carbon spectra of these samples revealed that the WTA signals could be divided into two fractions. The most rigid part of the WTA signal is present in ${ }^{1} \mathrm{H}$ to ${ }^{13} \mathrm{C}$ cross-polarization transfer experiments. As compared to the peptidoglycan resonances, it requires, however, longer $\mathrm{CP}$ contact times (several milliseconds against $250 \mu \mathrm{s}$ for PG resonances) to achieve a reasonable intensity (see as an example the $\mathrm{C} 1$ region of the ${ }^{13} \mathrm{C}$ spectrum in Figure 5b,c and Figure S2). This is the result of a partial averaging of the dipolar couplings in this part of the WTA as a result of molecular dynamics in the $\mathrm{kHz}$ range. The second part of the WTA signal reveals an even higher mobility with a complete averaging of all dipolar couplings. This part only appears in direct ${ }^{13} \mathrm{C}$ excited spectra in the form of narrow signals with completely resolved one-bond ${ }^{13} \mathrm{C}-{ }^{13} \mathrm{C}$ $J$-coupling (Figure 5a). In the absence of proton decoupling, the ${ }^{1} \mathrm{H}-{ }^{13} \mathrm{C} \mathrm{J}$-coupling can be resolved as well. Such a resolution can only be the result of extremely high dynamics of the chains. As the samples have been centrifuged and extensively rinsed several times, these high resolved signals cannot come from degraded (i.e., soluble) small WTA fragments. By comparing the equilibrium intensities of peptidoglycan and WTA resonances obtained in a $\mathrm{CP}$ build-up experiment and the ones measured by direct ${ }^{13} \mathrm{C}$ excitation (Figure S2), the fraction of WTA presenting a very fast dynamic behavior could be estimated to approximately $50 \%$. It is at first sight astonishing

(20) Kubler-Kielb, J.; Coxon, B.; Schneerson, R. J. Bacteriol. 2004, 186, 6891-6901. (a)

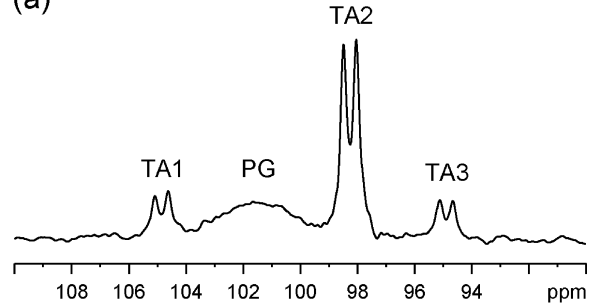

(b)

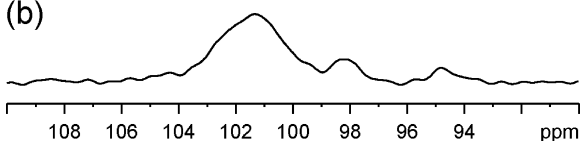

(c)

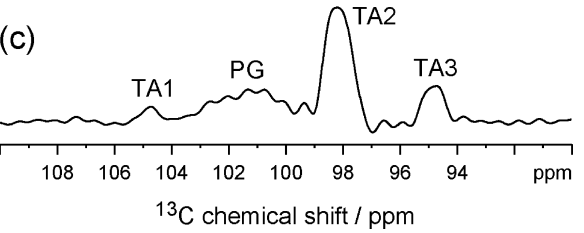

Figure 5. Comparison of the sugar $\mathrm{C} 1$ region of ${ }^{13} \mathrm{C}$ spectra recorded on the cell wall of B. subtilis 168 using (a) direct excitation, (b) $250 \mu \mathrm{s} \mathrm{CP}$ transfer, and (c) $4.5 \mathrm{~ms} \mathrm{CP}$ transfer experiments. This region is representative of what is observed in the entire spectrum. Spectra were recorded at 400 $\mathrm{MHz}$, and acquisition times were set to $18 \mathrm{~ms}$ for the $\mathrm{CP}$ experiments and $50 \mathrm{~ms}$ for the direct carbon excited spectrum. Line broadening of $20 \mathrm{~Hz}$ was applied to all spectra.

that these two populations of WTA with different dynamics are not detected in longitudinal ${ }^{13} \mathrm{C}$ or ${ }^{31} \mathrm{P}$ relaxation experiments, which revealed essentially a monoexponential behavior of magnetization (data not shown). This is explained by the fact that both techniques are sensitive to motional averaging but on different time scales: in the $\mathrm{MHz}$ range for the $T_{1}$ relaxation experiments and in the $\mathrm{kHz}$ range for the averaging of dipolar couplings as involved in $\mathrm{CP}$ experiments.

To characterize a possible alteration of the WTAs due to the preparation of cell wall samples, solid-state ${ }^{31} \mathrm{P}$ 1D NMR experiments were acquired on intact bacterial cells and on the purified sacculi in identical experimental conditions. In fact, ${ }^{31} \mathrm{P}$ chemical shifts are very sensitive to chemical substitutions in 

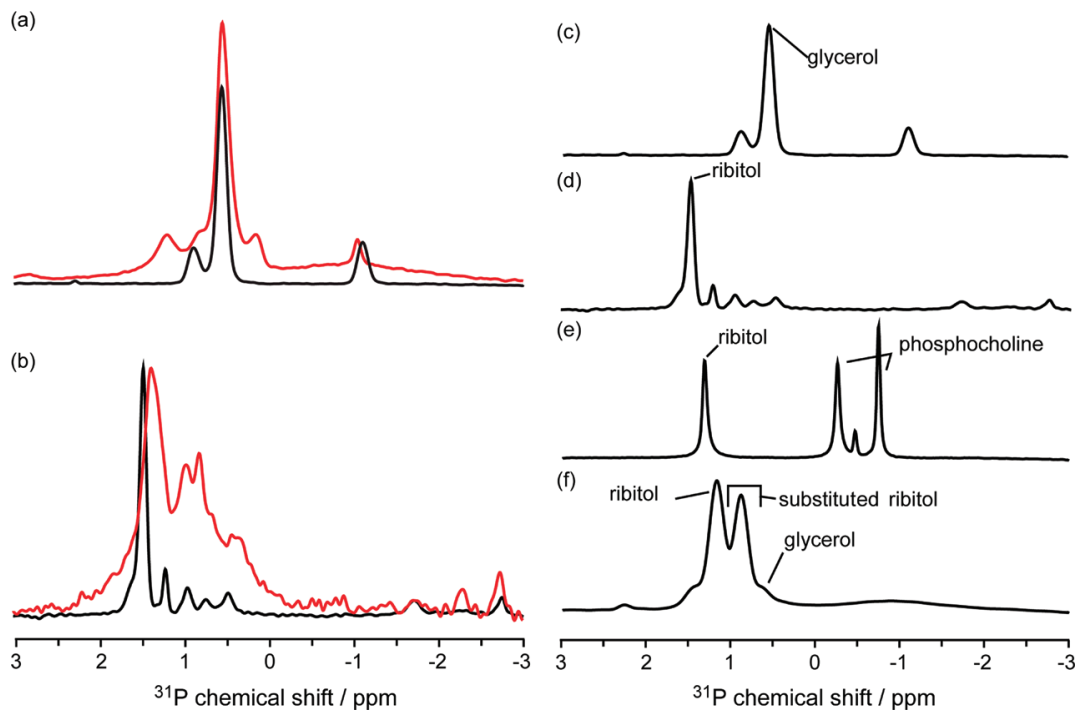

Figure 6. ${ }^{31} \mathrm{P}$ spectra of intact Gram-positive bacteria and cell wall samples. Left panel: comparison of direct excitation ${ }^{31} \mathrm{P}$ spectra recorded on intact cells (red) and isolated cell wall samples (black) from (a) B. subtilis 168 and (b) B. subtilis W23. Right panel: direct excitation ${ }^{31} \mathrm{P}$ spectra collected on isolated cell wall samples of (c) B. subtilis 168, (d) B. subtilis W23, (e) S. pneumoniae R6, and (f) S. aureus. The assignment of the different resonances is given in the figure.

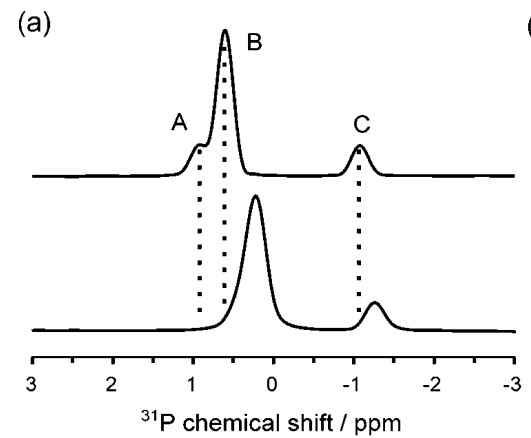

(b)

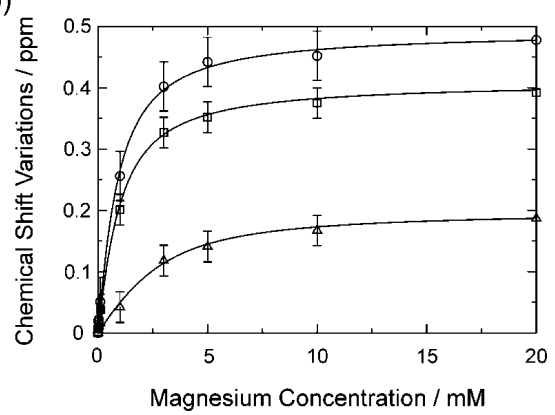

Figure 7. Quantitative analysis of the interaction of magnesium ions with the phosphate groups of the WTA from B. subtilis 168. Panel (a) shows the 1D ${ }^{31} \mathrm{P}$ spectra in the absence of magnesium (top trace) and in the presence of $20 \mathrm{mM} \mathrm{MgCl} 2$ (bottom trace). Panel (b) shows the ${ }^{31} \mathrm{P}$ chemical shift variations extracted from the $1 \mathrm{D}{ }^{31} \mathrm{P}$ spectra as a function of magnesium ion concentration for each individual ${ }^{31} \mathrm{P}$ resonance: peaks $\mathrm{A}(\mathrm{O})$, B $(\square)$, and C $(\triangle)$. Curve fitting yielded $K_{\mathrm{d}}$ values of $(595 \pm 232) \mu \mathrm{M}$ for peak A, $(686 \pm 321) \mu \mathrm{M}$ for peak B, and $(1242 \pm 730) \mu \mathrm{M}$ for peak C.

the vicinity of the phosphorus atom and to a slightly lesser extent to $\mathrm{pH}$ and/or ionic strength. The ${ }^{31} \mathrm{P}$ resonances detected for isolated cell wall samples and assigned to WTAs are also observed on intact bacterial sample at almost identical chemical shifts (see Figure 6a,b). This shows that the chemical structure of WTAs is unaffected by the treatment used for cell wall purification and allows the identification of WTA-specific resonances directly in complete bacteria, despite the presence of extra ${ }^{31} \mathrm{P}$ resonances. Thus, WTAs can be directly studied by solid-state NMR on intact bacterial cells or cell wall preparations. The interspecies comparison of ${ }^{31} \mathrm{P}$ spectra from isolated cell wall samples shown in Figure $6 \mathrm{c}-\mathrm{f}$ emphasizes the potential of solid-state NMR in the differentiation of WTA characteristics. By comparing NMR spectra of B. subtilis 168 , B. subtilis $\mathrm{W} 23, S$. pneumoniae, and $S$. aureus with data published for LTAs ${ }^{21-23}$ that bear phosphate groups similar to WTAs, partial assignment of the WTAs NMR spectra could be obtained.

(21) Klein, R. A.; Hartmann, R.; Egge, H.; Behr, T.; Fischer, W. Carbohydr. Res. 1996, 281, 79-98.

(22) Batley, M.; Redmond, J.; Wicken, A. Biochim. Biophys. Acta 1987, 901, 127-137.

(23) Roberts, M.; Jacobson, G.; Scott, P.; Mimura, C.; Stinson, M. Biochim. Biophys. Acta 1985, 845, 242-248.
Metal Binding to the WTAs. One of the functions attributed to teichoic acids is a role in ion trafficking. To analyze the interaction of metal ions with WTAs in the presence of the peptidoglycan, we used the very sensitive ${ }^{31} \mathrm{P}$ NMR probe. Figure $7 \mathrm{a}$ shows that the addition of $\mathrm{Mg}^{2+}$ ions produces chemical-shift variations of the WTA ${ }^{31} \mathrm{P}$ resonances. As the chemical shift variations appeared to be dependent on the concentration of $\mathrm{MgCl}_{2}$, further samples of $B$. subtilis cell walls were titrated with increasing concentrations of $\mathrm{Mg}^{2+}$ (Figure 7b). The effect of $\mathrm{Mg}^{2+}$ interaction on WTA flexibility was investigated by measuring ${ }^{31} \mathrm{P} T_{1}$ longitudinal relaxation timeconstants in the absence and in the presence of an excess (20 $\mathrm{mM}$ ) of divalent ions (Figure S3c). The very small increase of the relaxation time-constants observed in the presence of $\mathrm{Mg}^{2+}$ could indicate a small decrease of the flexibility of WTA induced by the interaction with the magnesium ions.

Similar effects could also be observed for manganese ions. Indeed, ${ }^{31} \mathrm{P}$ NMR spectra acquired on B. subtilis 168 cell wall samples, which were grown in the presence of $\mathrm{Mn}^{2+}$ ions and not treated with EDTA, showed significant line broadening of the ${ }^{31} \mathrm{P}$ WTA resonances as compared to EDTA-treated samples (data not shown). This broadening could only be explained by an interaction between the manganese paramagnetic ions and 


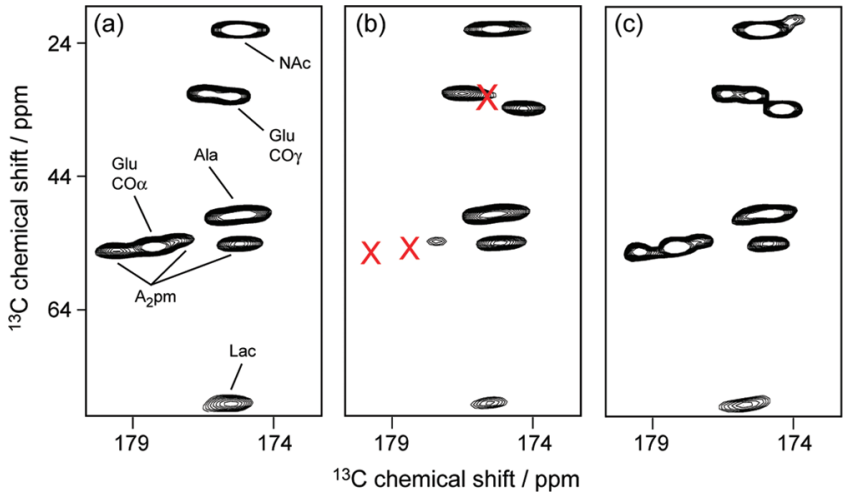

Figure 8. $\mathrm{Mn}^{2+}$-induced broadening effects observed on the ${ }^{13} \mathrm{C}$ peptidoglycan resonances of $B$. subtilis 168 cell wall using ${ }^{13} \mathrm{C}-{ }^{13} \mathrm{C}$ correlation experiments collected on a $400 \mathrm{MHz}$ Bruker spectrometer. The correlation region between carbonyl groups and aliphatic carbons is shown for peptidoglycan samples prepared (a) without WTA and without EDTA treatment, (b) with WTA and before EDTA treatment, and (c) with WTA and after EDTA treatment. Carbonyl correlations of the Glu and meso$\mathrm{A}_{2} \mathrm{pm}$ residues are broadened by the interaction with $\mathrm{Mn}^{2+}$ ions only in the presence of WTAs.

the WTA phosphate groups. $\mathrm{Mn}^{2+}$ ions were present in the growth medium $\left(0.1 \mathrm{mM} \mathrm{MnCl}_{2}\right)$, and they were not removed from the cell wall by several washing steps. Only a treatment with EDTA, which forms complexes with divalent ions with very low dissociation constants $\left(1.62 \mathrm{nM}\right.$ for $\mathrm{Mg}^{2+}$ and 13.49 $\mathrm{fM}$ for $\mathrm{Mn}^{2+}$ ), was able to remove the metal ions from the cell wall and to allow the recovery of a sharp ${ }^{31} \mathrm{P}$ line width. Nevertheless, it was impossible to obtain further information about the nature (electrostatic versus complexation) and location of the interaction site using ${ }^{31} \mathrm{P} \mathrm{NMR}$, although the more abundant glycerolphosphate groups seem to be the more affected. To learn more about the interaction, ${ }^{13} \mathrm{C}$ correlation experiments were performed under different conditions. As a result, in the presence of $\mathrm{Mn}^{2+},{ }^{13} \mathrm{C} \mathrm{CO}$-to- $\mathrm{C}_{\alpha}$ or CO-to- $\mathrm{C}_{\gamma}$ correlations of the glutamic acid and meso- $\mathrm{A}_{2} \mathrm{pm}$ carboxyl groups were significantly broadened such that they essentially disappeared from the ${ }^{13} \mathrm{C}-{ }^{13} \mathrm{C}$ through-bond correlation spectrum (Figure 8a,b). Interestingly, the interaction between the peptidoglycan and manganese ions only occurs significantly in the presence of WTAs (Figure $8 \mathrm{a}$ and $\mathrm{c}$ ). The concomitant resonance broadening observed in ${ }^{13} \mathrm{C}$ and ${ }^{31} \mathrm{P}$ spectra thus suggests a close proximity of the paramagnetic $\mathrm{Mn}^{2+}$ ions to the glutamic acid and to the meso- $\mathrm{A}_{2} \mathrm{pm}$ carboxyl groups of the peptidoglycan on one side, and to the phosphate groups of the WTAs on the other side (Figure 9).

\section{Discussion}

Properties of Peptidoglycan from Different Species. We have obtained NMR spectra from thick $B$. subtilis peptidoglycan sacculi, which were highly similar to spectra from thin $E$. coli sacculi (Figure 4a). The similarity of the NMR signals shows that the local organization of the two peptidoglycan samples is similar and is consistent with the related chemical composition of peptidoglycan in both species. Considering the distance between glycan strands, which is greater than $30 \AA$ (based on the length of the stem-peptides), we expect that the ${ }^{13} \mathrm{C}$ chemical shifts are mainly influenced by the local structure inside one glycan or peptide chain rather than by the presence of neighboring chains. Thus, as observed in this experiment, the presence of multiple peptidoglycan layers in Gram-positive species is not expected to affect the chemical shifts of the ${ }^{13} \mathrm{C}$ resonances.
Peptidoglycans from E. coli and B. subtilis differ mainly by their thickness ( $\sim 5$ and $\sim 30 \mathrm{~nm}$, respectively) and their average glycan strand length ( $\sim 30$ and more than 500 disaccharide units, respectively). ${ }^{3-8}$ The peptidoglycan of $S$. aureus is characterized by very short glycan strands (in average $\sim 5-10$ disaccharide units) ${ }^{24}$ and an unusually high degree of peptide cross-links, with the presence of multimeric peptides (up to 15-mers) (Figure $2 \mathrm{a}-\mathrm{c}) .9,25$ Our measurements of carbon longitudinal relaxation time-constants $T_{1}$ (Figure $4 \mathrm{~b}$ ) point out a global decrease of the molecular flexibility of the peptidoglycan in the following order: E. coli $>$ B. subtilis $>S$. aureus, the peptidoglycan of $S$. aureus being the most rigid of the three peptidoglycans studied in this Article. This finding is surprising and maybe counterintuitive. Indeed, the glycan strands, which are the more rigid part of the peptidoglycan, are shorter in $S$. aureus. In addition, the presence of the pentaglycine peptide bridge would be expected to enhance the flexibility as well. Obviously, the particularly high degree of peptide cross-linkage overcompensates for the shortness of the glycan strands and the presence of the peptide bridges and causes a major decrease in the overall flexibility of the $S$. aureus peptidoglycan (Figure 2d). Remarkably, comparison of the different ${ }^{13} \mathrm{C}$ resonances of the peptide stem revealed a relatively high local flexibility for the glycine residues, which, however, is not propagated to the rest of the peptide chain that remains more rigid in $S$. aureus than in B. subtilis or E. coli, where there is no interpeptide bridge. This finding shows that the presence of the interpeptide bridge cannot enhance flexibility in peptidoglycan with a high degree of peptide cross-linkage. An estimation of the change in peptidoglycan dynamics across the different strains can be obtained by comparing the variation in $T_{1}$ relaxation time-constants with their temperature dependence (Figure S1). Thus, the increase in dynamics observed between the peptidoglycan of $S$. aureus and E. coli can globally be compared to a temperature jump of about $30 \mathrm{~K}$.

Characterization of Wall Teichoic Acids. The behavior of ${ }^{13} \mathrm{C}$ WTA resonances with respect to proton decoupling and CP dynamics suggests that a significant portion of the WTAs is restricted in its dynamics by the peptidoglycan network. Although accurate determination of the flexibility of WTA was not possible due to multiple overlaps in the $1 \mathrm{D}$ spectrum, this finding indicates that the second portion of the teichoic acid chains is localized outside the peptidoglycan network. This interpretation of the data is supported by cryo-EM images of whole cells or thin sections, which suggest a "furry" surface on the cell that was supposed to be the wall- or lipid-linked TAs. ${ }^{26}$

WTAs and LTAs as well as degraded fragments from various bacterial species have been previously investigated by ${ }^{31} \mathrm{P}$ liquidstate NMR spectroscopy. ${ }^{21-23,27}$ The only previous solid-state NMR study of TA analyzed the interaction of dried LTA with different surfaces at low MAS speed. ${ }^{28,29}$ We show here that performing ${ }^{31} \mathrm{P}$ experiments on hydrated cell walls or intact bacterial samples at a reasonable MAS speed $(\sim 10 \mathrm{kHz})$ provides solid-state NMR spectra of a resolution similar to that found in liquid-state NMR. The advantage of the ${ }^{31} \mathrm{P}$ nucleus

(24) Boneca, I. G.; Huang, Z. H.; Gage, D. A.; Tomasz, A. J. Biol. Chem. 2000, 275, 9910-9918.

(25) Snowden, M. A.; Perkins, H. R. Eur. J. Biochem. 1990, 191, 373377.

(26) Matias, V.; Beveridge, T. Mol. Microbiol. 2005, 56, 240-251.

(27) Schipper, D. Carbohydr. Res. 1995, 279, 75-82.

(28) Rice, C. V.; Wickham, J. R. J. Am. Chem. Soc. 2005, 127, 856-857.

(29) Wickham, J.; Rice, C. Solid State Nucl. Magn. Reson. 2008, 34, 154161. 


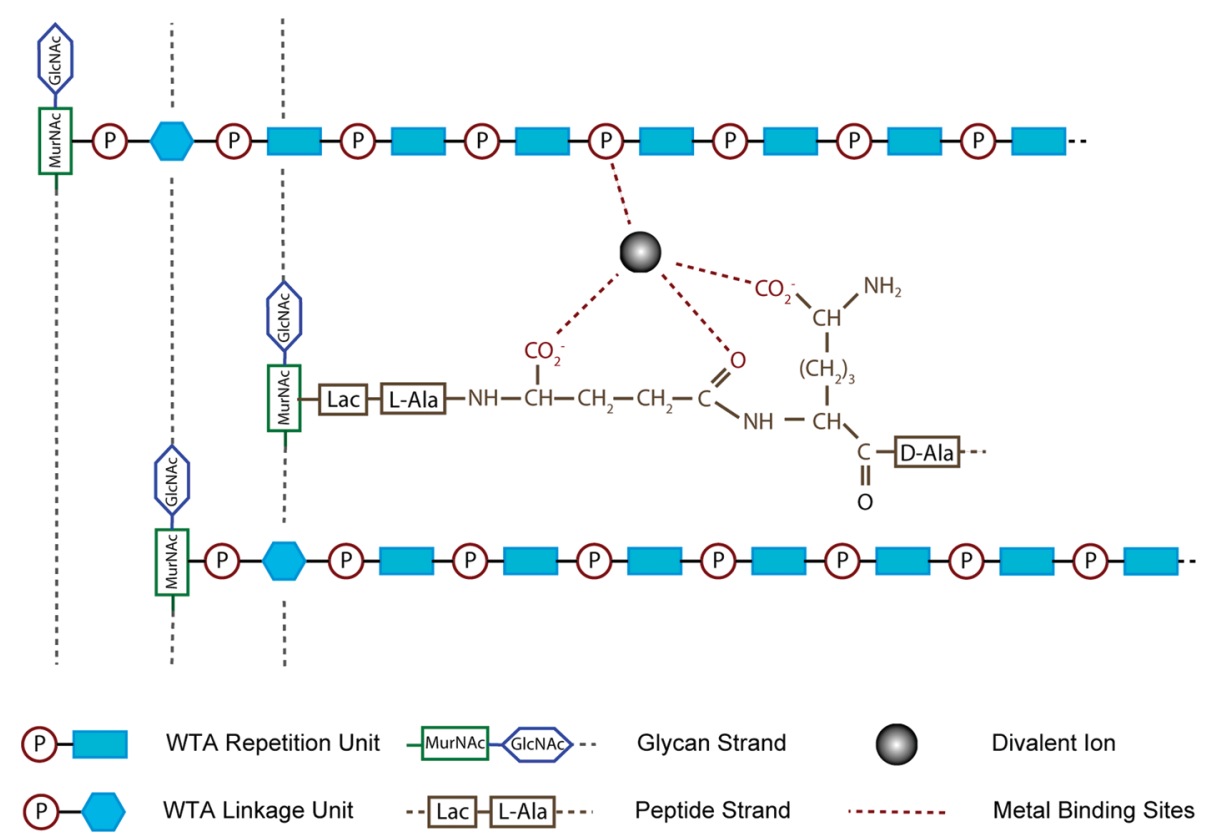

Figure 9. Schematic representation of possible binding sites for divalent metal ions on the example of the cell wall of B. subtilis. Red dotted lines show cooperative metal binding between the peptide strands of the peptidoglycan and the phosphate groups of the WTA. Lines from the carboxylate or the phosphate groups could represent either monodentate or bidentate ligation modes.

over the ${ }^{13} \mathrm{C}$ nucleus is its $100 \%$ natural abundance (vs $1.1 \%$ only for ${ }^{13} \mathrm{C}$ ) as well as its appreciable sensitivity (4.2 times that of ${ }^{13} \mathrm{C}$ ), making solid-state ${ }^{31} \mathrm{P}$ NMR an easy and quick tool for further investigations of cell walls of Gram-positive species without the need of isotopic labeling. The comparative study on a variety of bacteria detailed in Figure 6 demonstrates that the rapid acquisition of a solid-state $1 \mathrm{D}^{31} \mathrm{P}$ NMR spectrum on hydrated intact bacteria (or processed cell walls with intact teichoic acids) allows easy identification of fine differences in the cell wall structure of bacterial species. The application of this method should provide a particularly powerful tool to determine the effect of external factors on the WTA biosynthesis and chemical modifications, as well as having relevance in species identification and typification. Bacterial cells remained viable during the acquisition of ${ }^{31} \mathrm{P}$ NMR spectra (MAS, RF irradiation...) and could be grown on agar plates after the NMR experiment.

Metal Binding to Wall Teichoic Acids. Interaction between anionic TAs (or negatively charged TAs) and cationic divalent ions has been demonstrated and largely discussed in the past. $^{22,29-32}$ Data obtained by dialysis exchange or liquid-state NMR on isolated LTAs indicated a relatively low affinity for $\mathrm{Mg}^{2+}\left(K_{\mathrm{d}} \approx 15 \mathrm{mM}\right)$ as well as for $\mathrm{Ca}^{2+}\left(K_{\mathrm{d}} \approx 8.9 \mathrm{mM}\right){ }^{32}$ The affinity of teichoic acids for $\mathrm{Mg}^{2+}$ is comparable to the one for free $\mathrm{HPO}_{4}{ }^{2-}$ ions. However, a lower dissociation constant of approximately $350 \mu \mathrm{M}$ has been measured on intact $S$. aureus cell wall, ${ }^{31}$ and the possibility of a bidentate coordination between wall teichoic acids and $\mathrm{Mg}^{2+}$ has been proposed. ${ }^{33,34}$

(30) Lambert, P.; Hancock, I.; Baddiley, J. Biochem. J. 1975, 149, 519524.

(31) Lambert, P.; Hancock, I.; Baddiley, J. Biochem. J. 1975, 151, 671676.

(32) Rose, R. K.; Hogg, S. D. Biochim. Biophys. Acta, Gen. Subj. 1995, 1245, 94-98.

(33) Heckels, J. E.; Lambert, P. A.; Baddiley, J. Biochem. J. 1977, 162, $359-365$.

(34) Wickham, J. R.; Halye, J. L.; Kashtanov, S.; Khandogin, J.; Rice, C. V. J. Phys. Chem. B 2009, 113, 2177-2183.
In our study, metal ion interactions within the complete cell wall (containing WTA and peptidoglycan) have been investigated using solid-state NMR. The analysis of the ${ }^{31} \mathrm{P}$ chemicalshift variations as a function of the magnesium concentration leads to a dissociation constant of about $600 \pm 300 \mu \mathrm{M}$ for the phosphate of the repeating unit in B. subtilis 168 (Figure 7). Thus, the interaction of $\mathrm{Mg}^{2+}$ with intact cell wall is about 25 times stronger than that with isolated TAs $\left(K_{\mathrm{d}} \approx 15 \mathrm{mM}\right)$, suggesting that the architectural arrangement of the WTA polymer in the cell wall could form an integrated system for binding of cations.

The presence of $\mathrm{Mn}^{2+}$ ions in cell wall samples was detected through line broadening of the corresponding ${ }^{31} \mathrm{P}$ and ${ }^{13} \mathrm{C} \mathrm{NMR}$ resonances, suggesting that manganese also interacts with the phosphate group of the TA. Interestingly, ${ }^{13} \mathrm{C}$ correlation spectra of the cell wall of B. subtilis taken directly after purification from a growth medium containing $0.1 \mathrm{mM} \mathrm{Mn}^{2+}$ ions without EDTA treatment showed the loss of the glutamic acid and meso$\mathrm{A}_{2} \mathrm{pm}$ carboxyl group resonances. Analyzing the ${ }^{13} \mathrm{C}$ and ${ }^{31} \mathrm{P}$ data together and taking into account that binding of $\mathrm{Mn}^{2+}$ ions does not occur significantly in the absence of WTAs, we propose an intermolecular complexation of the $\mathrm{Mn}^{2+}$ ion by the carboxyl groups of D-Glu and meso- $\mathrm{A}_{2} \mathrm{pm}$ of the peptidoglycan and by the phosphate groups of the teichoic acids (Figure 9). The $\mathrm{Mn}^{2+}$ metal center is usually found in an octahedral geometry with oxygen donor ligands. The peptidoglycan carboxylate groups from $\gamma$-D-Glu and meso- $\mathrm{A}_{2}$ pm can provide a bis $\left(\eta^{2}\right.$-carboxylato) scaffold for the manganese complexation, which can be perfectly complemented with a mixture of monodentate phosphate groups of the teichoic acids repetition units and of water molecules or alternatively by a bidentate phosphate ligand. ${ }^{34}$ Taken together, these results provide the first experimental evidence for WTA and peptidoglycan acting in a cooperative role in the geometry and the thermodynamics of the binding to divalent cations. It also gives an explanation for the lower dissociation constant of $\mathrm{Mg}^{2+}$ binding to the cell wall when compared to isolated TAs. 
Amidation of carboxyl groups prevents interaction with metal ions. In B. subtilis, the amidation of the carboxyl group of $\mathrm{A}_{2} \mathrm{pm}$ is modulated as a function of the growth conditions and thus could represent a mechanism to regulate the amount of cell wall bound ions. For $S$. aureus, where the glutamic acid is always amidated, $\mathrm{Mg}^{2+}$ binding might be controlled by the rate of alanyl substitution in the TAs as discussed in the literature. ${ }^{31,35}$ Inverse correlation between the rate of alanylation and $\mathrm{Mg}^{2+}$ concentration has been determined for different growth conditions. ${ }^{36,37}$ The proposed peptidoglycan-WTA mediated binding of cations could be a key factor for storage and supply of cations essential for bacterial growth. ${ }^{38}$ Divalent cations are known to be required for growth and survival of certain mutant strains of $B$. subtilis. ${ }^{38-40}$ For example, a mutant lacking functional MreB, a cytoskeletal protein governing cell wall elongation, loses rodshape and viability at normal conditions but grows normally if the concentration of $\mathrm{Mg}^{2+}$ ions is increased to $10 \mathrm{mM} \cdot{ }^{39}$ These mutants with impaired mode of cell wall synthesis or cell wall structure appear to require the stabilizing effect of cell wall bound cations, which we here show are capable of forming multiple sites of interactions between PG and WTA. It has also been assumed that the isoelectric point of the cell wall determines the susceptibility of the cell to cationic antimicrobial host defense molecules, bacteriocins, and antibiotics ${ }^{13}$ and could play a role in cell adhesion. ${ }^{41}$ Cation-mediated interactions between peptidoglycan and WTA, as described here, could represent an important structural aspect of Gram-positive cell wall architecture. It has been proposed that for Gram-positive bacteria, the absence of an external membrane is compensated by a thicker peptidoglycan whose porosity is reduced by the TAs. ${ }^{14}$

\section{Conclusion}

Despite decades of research, the structure of bacterial cell walls has remained a challenging topic due to the enormous size and complex heterogeneous composition of these biomolecules. In this work, we show that it is possible to obtain surprisingly well-resolved solid-state ${ }^{13} \mathrm{C}-{ }^{13} \mathrm{C}$ NMR correlation spectra from hydrated cell wall samples of a range of different species. On the basis of our previous experience with sacculi from $E$. coli ${ }^{19}$ we present here solid-state NMR data from cell walls and even intact cells of Gram-positive species. These data provide novel and important insights into the flexibility and molecular arrangements of the two major cell wall components in Gram-positive bacteria.

Thus, we have shown that organization and flexibility of different bacterial cell walls can be analyzed by ${ }^{13} \mathrm{C}$ and ${ }^{31} \mathrm{P}$ solid-state NMR. The comparison of carbon correlation spectra and relaxation experiments has shown that, despite a similar structural architecture, the flexibility of the peptidoglycan decreases going from the Gram-negative E. coli to the Grampositive B. subtilis and even more for S. aureus, another Gram-

(35) Neuhaus, F.; Baddiley, J. Microbiol. Mol. Biol. Rev. 2003, 67, 686723.

(36) Heptinstall, S.; Archibald, A.; Baddiley, J. Nature 1970, 225, 519521.

(37) Hurst, A.; Hughes, A.; Duckworth, M.; Baddiley, J. J. Gen. Microbiol. 1975, 89, 277-284.

(38) Schirner, K.; Marles-Wright, J.; Lewis, R. J.; Errington, J. EMBO J. 2009, 28, 830-842.

(39) Formstone, A.; Errington, J. Mol. Microbiol. 2005, 55, 1646-1657.

(40) Kawai, Y.; Daniel, R. A.; Errington, J. Mol. Microbiol. 2009, 71, 1131 1144.

(41) Rijnaarts, H. H. M.; Norde, W.; Lyklema, J.; Zehnder, A. J. B. Colloids Surf., $B$ 1995, 4, 191-197. positive bacteria. The decrease in dynamics is shown to be governed by the degree of cross-linkage, which compensates for other structural parameters like short glycan strands or the presence of interpeptide bridges. By comparing the NMR relaxation behavior of ${ }^{13} \mathrm{C}$ and ${ }^{31} \mathrm{P}$ nuclei, local flexibility can thus be analyzed and correlated to other physical properties. This strategy will permit the characterization of other specific cell wall properties, for example, in the presence of an antibiotic stress.

${ }^{31} \mathrm{P}$ solid-state NMR is demonstrated to be a powerful tool for the study of wall teichoic acids in isolated cell walls as well as in intact cell samples, as it is a very sensitive nucleus for NMR and does not require any specific labeling. Thus, changes in the chemical structure of WTA are very rapidly detected in both isolated cell wall and intact cell samples, as demonstrated for example on two different species of B. subtilis. The potential utility of ${ }^{31} \mathrm{P}$ solid-state NMR in species identification and typification is enlightened. ${ }^{31} \mathrm{P}$ solid-state NMR is also shown to be particularly sensitive to the presence of divalent metal ions and affords a way to efficiently determine the binding constant. Complemented with ${ }^{13} \mathrm{C}$ NMR on uniformly labeled samples, it allows the identification of the binding sites. Thus, the combination of ${ }^{13} \mathrm{C}$ and ${ }^{31} \mathrm{P}$ solid-state NMR puts in evidence that divalent ions are stabilized in the cell wall by both the peptidoglycan and the WTA. This information allowed us to propose a new geometry for the coordination of different divalent metal ions.

\section{Materials and Methods}

Cell Wall Preparation. Cell wall samples from B. subtilis and $S$. aureus were uniformly ${ }^{13} \mathrm{C}$ - and ${ }^{15} \mathrm{~N}$-isotopically labeled. Therefore, Bacillus subtilis subsp. subtilis strain 168 cells from the American Collection (ATCC 23857) were grown in standard ${ }^{13} \mathrm{C},{ }^{15} \mathrm{~N}$-labeled M9 medium containing $0.1 \mathrm{mM} \mathrm{MnCl}_{2}$, and Staphylococcus aureus (ATCC 25923) cells were grown in a ${ }^{13} \mathrm{C},{ }^{15} \mathrm{~N}$-SILANTES medium (www.silantes.com). Bacillus subtilis subsp. spizizenii W23 was grown in standard LB medium, and Streptococcus pneumoniae R6 was grown in $\mathrm{C}+\mathrm{Y}$ medium. ${ }^{42}$ Cells were harvested by centrifugation at an OD $(600 \mathrm{~nm})$ of about 0.7 and boiled in 4\% SDS. Detergent was eliminated with milli-Q water. Highly purified cell walls were obtained by a previously published procedure. ${ }^{43,44}$ This procedure retained the intact cell envelope (sacculi) formed by the peptidoglycan and covalently linked WTAs, while LTAs were eliminated through the SDS treatment of the bacterial cells in the isolation steps. ${ }^{26,45}$ Additional hydrofluoric acid treatment of the cell wall removed the WTA by hydrolysis of the phosphate group attached to position 6 of the $N$-acetylmuramic acid. Thus, hydrated uniformly ${ }^{13} \mathrm{C}$ - and ${ }^{15} \mathrm{~N}$-labeled peptidoglycan samples, with and without attached WTAs, were obtained from $B$. subtilis and $S$. aureus.

NMR Sample Preparation. Peptidoglycan. The purified peptidoglycan was spun down by ultracentrifugation and resuspended in a $50 \mathrm{mM}$ HEPES buffer at $\mathrm{pH} 7.5$. These steps were repeated until the $\mathrm{pH}$ of the supernatant remained stable at 7.5. A hydrated gel-like suspension of peptidoglycan was packed in the rotor by centrifugation.

Cell Wall. Lyophilized cell walls were resuspended in HEPES buffer $\mathrm{pH} 7.5$ and were treated in the same manner as the

(42) Horne, D. S.; Tomasz, A. J. Bacteriol. 1993, 175, 1717-1722.

(43) Severin, A.; Tomasz, A. J. Bacteriol. 1996, 178, 168-174.

(44) Girardin, S. E.; Boneca, I. G.; Carneiro, L. A. M.; Antignac, A.; Jéhanno, M.; Viala, J.; Tedin, K.; Taha, M.; Labigne, A.; Zähringer, U.; Coyle, A. J.; DiStefano, P. S.; Bertin, J.; Sansonetti, P. J.; Philpott, D. J. Science 2003, 300, 1584-1587.

(45) Birdsell, D.; Doyle, R.; Morgenstern, M. J. Bacteriol. 1975, 121, 726734. 
peptidoglycan. Complete removal of metal ions for reference peptidoglycan and cell wall samples was achieved by a $500 \mathrm{mM}$ EDTA treatment before conditioning of the samples in HEPES (metal-free) buffer.

Samples for $\mathbf{M g}^{2+}$ Titration Experiments. A metal ion-free cell wall sample of $B$. subtilis 168 (ATCC 23857) was separated into 10 equivalent fractions, which where then spun down. The pellets were resuspended in $1 \mathrm{~mL}$ of $50 \mathrm{mM}$ HEPES buffer $\mathrm{pH} 7.5$ containing different concentrations of $\mathrm{Mg}^{2+}(0,10 \mu \mathrm{M}, 20 \mu \mathrm{M}, 30$ $\mu \mathrm{M}, 100 \mu \mathrm{M}, 1 \mathrm{mM}, 3 \mathrm{mM}, 5 \mathrm{mM}, 10 \mathrm{mM}, 20 \mathrm{mM})$ and $1 \%$ HMPA for NMR spectra referencing. The overall ${ }^{31} \mathrm{P}$ concentration of the sample that is used in the fitting equation was experimentally estimated by integration of the ${ }^{31} \mathrm{P}$ signals in the $1 \mathrm{D}$ NMR spectrum and comparison with ${ }^{31} \mathrm{P}$ signals of HMPA used as an internal standard. The samples at 0 and $20 \mathrm{mM} \mathrm{Mg}^{2+}$ concentrations were also used for ${ }^{31} \mathrm{P}$ longitudinal relaxation measurements.

Solid-State NMR Experiments. NMR experiments were performed at 9.4 T ( ${ }^{1} \mathrm{H}$ NMR frequency at $400.13 \mathrm{MHz}$ ) using a Bruker $400 \mathrm{MHz}$ spectrometer equipped with a Bruker $4 \mathrm{~mm}$ tripleresonance probe and at $14.1 \mathrm{~T}\left({ }^{1} \mathrm{H} \mathrm{NMR}\right.$ frequency at $\left.600 \mathrm{MHz}\right)$ using a Varian BioMAS spectrometer equipped with a $3.2 \mathrm{~mm}$ triple resonance probe. For the Bruker spectrometer, the MAS spinning speed was set to $9 \mathrm{kHz}$ and the bearing gas was stabilized at a temperature of $268 \mathrm{~K}$, corresponding to a sample temperature of $283 \mathrm{~K}$. The experiments on the Varian spectrometer were done with a MAS spinning speed set to $12.5 \mathrm{kHz}$ and a sample temperature of $285 \mathrm{~K}$.

${ }^{31} \mathrm{P}$ 1D NMR spectra were obtained by direct ${ }^{31} \mathrm{P}$ excitation. For the spectra acquired at $400 \mathrm{MHz}$, the phosphorus rf-field strength was set to $60 \mathrm{kHz}$. During acquisition, SPINAL or CW heteronuclear decoupling was used at a proton rf-field strength of 90 or $45 \mathrm{kHz}$, respectively. Acquisition time was between 50 and 120 $\mathrm{ms}$, depending on the decoupling field strength. The experimental time was $1 \mathrm{~h}$ with an interscan delay of $5 \mathrm{~s}$.

For the $\mathrm{Mg}^{2+}$ binding experiments done on the $600 \mathrm{MHz}$ spectrometer, the spectral width was set to $10 \mathrm{kHz}$. The acquisition time was $20 \mathrm{~ms}$, and the interscan delay was $1 \mathrm{~s}$. Experimental time was about $15 \mathrm{~min}$ per titration point. ${ }^{31} \mathrm{P}$ longitudinal relaxation time-constants were measured by inversion recovery after direct excitation. The interscan delay in the later experiments was increased to $5 \mathrm{~s}$. During acquisition, TPPM heteronuclear decoupling was used at a proton rf-field strength of $100 \mathrm{kHz}$, and the phosphorus rf-field strength was set to $50 \mathrm{kHz}$. To determine the affinity of $\mathrm{Mg}^{2+}$ for $B$. subtilis cell walls from the experimental data, the ${ }^{31} \mathrm{P}$ chemical-shift variations were measured as a function of the $\mathrm{Mg}^{2+}$ concentration. Curves of chemical-shift variations as a function of $\mathrm{Mg}^{2+}$ concentration have been obtained for the different ${ }^{31} \mathrm{P}$ resonances and plotted. Dissociation constants were extracted by fitting the curves independently using the classical 1:1 binding equation, ${ }^{46}$ considering that each phosphate repeating unit can interact with one magnesium ion.

(46) Boudet, J.; Duval, V.; Van Melckebeke, H.; Blackledge, M.; Amoroso, A.; Joris, B.; Simorre, J. Nucleic Acids Res. 2007, 35, 4384-4395.
The ${ }^{13} \mathrm{C}$ through-bond correlation experiments were performed at $400 \mathrm{MHz}$ as already published ${ }^{19}$ except that initial ${ }^{13} \mathrm{C}$ magnetization was obtained by an adiabatic cross-polarization step of 500 $\mu \mathrm{s},{ }^{47}$ performed at an averaged rf-field strength of $69 \mathrm{kHz}$ on ${ }^{1} \mathrm{H}$ and $60 \mathrm{kHz}$ on ${ }^{13} \mathrm{C}$. For ${ }^{1} \mathrm{H}$ pulses and SPINAL heteronuclear decoupling ${ }^{48}$ during $J$-evolution and acquisition, the rf-field strength was set to $90 \mathrm{kHz}$. The $J$-evolution periods were rotor synchronized and set to $2 \mathrm{~ms}$, except for experiments on $E$. coli where it was set to $1.667 \mathrm{~ms}$. The spectral width was $24 \mathrm{kHz}$ in both dimensions. Acquisition times were $15-18 \mathrm{~ms}$ in the direct dimension, and 5-7 $\mathrm{ms}$ in the indirect dimension using States-TPPI quadrature detection. The interscan delay was set to $1.5 \mathrm{~s}$ for E. coli and S. aureus, and to $2 \mathrm{~s}$ for $B$. subtilis, leading to a total experimental time between 18 and $62 \mathrm{~h}$ depending on the bacterial strain. Carbon longitudinal relaxation time-constants were measured by inversion-recovery after direct excitation of carbons. The experiments were reproduced on two independent samples of E. coli and B. subtilis sacculi to control the reproducibility of the results. The direct excitation of carbons has been preferred to cross-polarization, which leads to a nonuniform distribution of magnetization on the different carbon sites and to a distortion of the first points in the decay curve. The interscan delay (4-10 s) was set at least to $5 \times$ the longest $T_{1}$ value. Other parameters were set as described above.

${ }^{13} \mathrm{C} 1 \mathrm{D}$ spectra acquired on the $600 \mathrm{MHz}$ spectrometer were recorded with a $40 \mathrm{kHz}$ spectral width. Acquisition time was 14 $\mathrm{ms}$, and the interscan delay was $5 \mathrm{~s}$. Rf-field strength was set to 46 $\mathrm{kHz}$ for ${ }^{13} \mathrm{C}$ and $90 \mathrm{kHz}$ for ${ }^{1} \mathrm{H}$. During acquisition, TPPM heteronuclear decoupling was used at a proton rf-field strength of $90 \mathrm{kHz}$. Carbon longitudinal relaxation time-constants as a function of temperature were measured by inversion-recovery after direct excitation of carbons.

Acknowledgment. This research was supported by the European Union FP6 EUR-INTAFAR (Project LSHM-CT-2004-512138) and the ANR (ANR-08-BLAN-0306-01 and -02). We thank Alice Eberhardt from Newcastle University for technical assistance in cell wall preparation and Richard Daniel for critical reading of the manuscript.

Supporting Information Available: Temperature dependence of ${ }^{13} \mathrm{C}$ and ${ }^{31} \mathrm{P} T_{1}$ relaxation time-constants, cross-polarization build-up curves for peptidoglycan and WTA resonances, $\mathrm{Mg}^{2+}$ effect on $1 \mathrm{D}{ }^{31} \mathrm{P}$ NMR spectrum and ${ }^{31} \mathrm{P} T_{1}$ relaxation timeconstants, and ${ }^{13} \mathrm{C}$ teichoic acids chemical shift assignments. This material is available free of charge via the Internet at http:// pubs.acs.org.

JA104533W

(47) Hediger, S.; Meier, B.; Ernst, R. Chem. Phys. Lett. 1995, 240, 449456.

(48) Fung, B.; Khitrin, A.; Ermolaev, K. J. Magn. Reson. 2000, 142, 97101. 